\title{
Prognostic association of cardiac anxiety with new cardiac events and mortality following myocardial infarction
}

Maria H. C. T. Van Beek, Marij Zuidersma, Martijn Lappenschaar, Gheorghe Pop, Annelieke M. Roest, Anton J. L. M. Van Balkom, Anne E. M. Speckens and Richard C. Oude Voshaar

\section{Background}

General anxiety and depressive symptoms following a myocardial infarction are associated with a worse cardiac prognosis. However, the contribution of specific aspects of anxiety within this context remains unclear.

\section{Aims \\ To evaluate the independent prognostic association of cardiac anxiety with cardiac outcome after myocardial infarction.}

\section{Method}

We administered the Cardiac Anxiety Questionnaire (CAQ) during hospital admission (baseline, $n=193$ ) and 4 months $(n=147 / 193)$ after discharge. CAQ subscale scores reflect fear, attention, avoidance and safety-seeking behaviour. Study end-point was a major adverse cardiac event (MACE): readmission for ischemic cardiac disease or all-cause mortality. In Cox regression analysis, we adjusted for age, cardiac disease severity and depressive symptoms.

\section{Results}

The CAQ sum score at baseline and at 4 months significantly predicted a MACE ( $\mathrm{HR}_{\text {baseline }}=1.59,95 \% \mathrm{Cl} 1.04-2.43$; $\left.\mathrm{HR}_{4 \text {-months }}=1.77,95 \% \mathrm{Cl} 1.04-3.02\right)$ with a mean follow-up of 4.2 (s.d. $=2.0$ ) years and $4.3($ s.d. $=1.7)$ years respectively. Analyses of subscale scores revealed that this effect was particularly driven by avoidance $\left(\mathrm{HR}_{\text {baseline }}=1.23,95 \% \mathrm{Cl}\right.$ 0.99-1.53; $\mathrm{HR}_{4 \text {-months }}=1.77,95 \% \mathrm{Cl} 1.04-1.83$ ).

\section{Conclusions}

Cardiac anxiety, particularly anxiety-related avoidance of exercise, is an important prognostic factor for a MACE in patients after myocardial infarction, independent of cardiac disease severity and depressive symptoms.

\section{Declaration of interest}

None.

\section{Copyright and usage}

(c) The Royal College of Psychiatrists 2016.
Potential factors that determine cardiac prognosis after a myocardial infarction include demographic and clinical parameters, health behaviours and psychiatric morbidities. Recently, the impact of anxiety symptoms on cardiac prognosis in patients with heart disease has gained more attention. In a meta-analysis, post-myocardial infarction anxiety was associated with a $36 \%$ increased risk of adverse cardiac outcomes. ${ }^{1}$ Although this meta-analysis could not adjust for measures of disease severity, effect estimates of included studies that did adjust for measures of disease severity were only slightly or not attenuated. ${ }^{1}$ Also, generalised anxiety disorder (GAD) was related to adverse outcomes in patients with myocardial infarction and this relationship was not explained by cardiac disease severity parameters. ${ }^{2}$ In contrast, other studies described no association ${ }^{3-5}$ or even a beneficial association in patients with myocardial infarction $^{6,7}$ and those with stable coronary artery disease $(\mathrm{CAD})^{8}$ between anxiety and cardiac prognosis. Furthermore, a recent study in patients with CAD undergoing coronary artery bypass graft surgery showed different associations of different types of anxiety with cardiac prognosis: no association of fear and panic disorders and their symptom dimensions, but an adverse association with GAD. ${ }^{9}$ These controversial findings suggest that different types of anxiety may be related differently to cardiac prognosis. As it remains unclear which specific aspects of anxiety are associated with cardiac prognosis, the need to examine the unique contribution of types of anxiety on cardiovascular risk has been advocated. ${ }^{1,10}$ Recently, a population-based study found that worry predicted non-fatal cardiac outcome over a 3-year follow-up, whereas panic and phobia did not. ${ }^{11}$ Another population-based study suggested that in women phobic anxiety may predict fatal but not non-fatal cardiac events. ${ }^{12}$
After a myocardial infarction, cardiac stimuli and sensations may trigger specific anxiety symptoms, conceptualised as cardiac anxiety. This can be assessed reliably by the Cardiac Anxiety Questionnaire (CAQ). ${ }^{13,14}$ CAQ subscale scores in patients with myocardial infarction reflect fear, attention, avoidance of physical exercise and safety-seeking behaviour. ${ }^{14}$ Higher cardiac anxiety is shown to be associated with lower quality of life in patients with myocardial infarction. ${ }^{15}$ To our knowledge, the association between cardiac anxiety and cardiac outcome has not been examined previously. Furthermore, in most studies anxiety symptoms are measured directly after hospital admission or surgery, ${ }^{1}$ which may inflate the rates of psychopathology as a result of the temporary distress of admission to hospital. Longterm functional outcome may only be affected by psychopathological symptoms that persist, or develop during the first weeks as has been demonstrated for depressive symptoms. ${ }^{16}$ Previously, we showed that trajectories of cardiac anxiety after a myocardial infarction are largely determined within the first 3-4 months after the event. ${ }^{15}$ The aim of the present study was to explore the impact of self-reported cardiac anxiety at both admission to hospital and at 4 months after discharge on cardiac prognosis after a myocardial infarction. We hypothesised that patients reporting elevated symptoms of cardiac anxiety had the worst cardiac prognosis independent of potential confounders like cardiac disease severity and depressive symptoms.

\section{Method}

\section{Study population}

For the present analysis we included patients with myocardial infarction enrolled in an exploratory prospective cohort study 
described previously. ${ }^{15}$ Patients consecutively admitted to hospital with myocardial infarction between November 2006 and December 2007 to the Department of Cardiology of Radboud University Medical Centre, The Netherlands, were recruited within 2 days of admission. Eligible patients had to be diagnosed with ST-elevated myocardial infarction (STEMI) or non-STelevated myocardial infarction (NSTEMI). The diagnosis was confirmed by the presence of a rise and fall of troponin I $(>0.20 \mu \mathrm{g} / \mathrm{L})$.

Exclusion criteria were: age above 85 years, discharge from hospital within 2 days of admission, and inability to fill out questionnaires (because of insufficient knowledge of the Dutch language, cognitive impairment or being too ill to participate). The study protocol was approved by the local medical ethics committee, and all patients provided written informed consent.

\section{Procedure}

Between days 2 and 7 after admission (baseline), patients completed a set of self-report questionnaires including the $\mathrm{CAQ}^{13,14}$ and the Beck Depression Inventory (BDI). ${ }^{17}$ Four months after discharge patients were sent the same questionnaires by post. If necessary, patients were contacted by phone as a reminder.

\section{Measures}

\section{Assessment of cardiac anxiety}

Cardiac anxiety was assessed with the 18 -item self-reportquestionnaire $\mathrm{CAQ}$, rating each item on a five-point Likert scale ranging from 0 (never) to 4 (always). ${ }^{13}$ In line with previous publications, the overall score as well as subscale scores are expressed as an average item score, which was computed by summing the score for the relevant items and dividing it by the number of items. The CAQ is well-validated in different populations, originally by Eifert et al. ${ }^{13,14,18}$ Recently, we crossvalidated the CAQ in patients with myocardial infarction and identified a factor structure of four subscales: assessing fear (for example, 'When I have chest discomfort, or when my heart is beating fast: I get frightened'), attention (for example, 'I pay attention to my heartbeat'), avoidance of (physical) activity (such as, 'I avoid activities that make my heart beat faster') and safety-seeking behaviour (such as, 'When I have chest discomfort or when my heart is beating fast, I like to be checked out by a doctor'), respectively. ${ }^{14}$ Our study showed a good internal consistency of both total and subscale scores (Cronbach $\alpha=0.84$ and $0.6-0.9$, respectively), a high test-retest-reliability $(0.88$, $P<0.001)$ and low to moderate correlations with questionnaires such as the Agoraphobic Cognitions questionnaire (0.31), the State-Trait- Anxiety Inventory (0.39) and the Beck Depression Inventory (BDI, 0.27). Comparable results, including the four subscales, have been previously reported in an independent cross-validation study in 658 people referred for screening for coronary artery disease. ${ }^{18}$ We confirmed the four subscales factor solution on the present data. In the present study, our main predictors were the continuous overall mean item score of the CAQ (i.e. total score divided by number of items), as well as the mean item score of the subscales.

\section{Assessment of covariates}

\section{Sociodemographic and cardiac-related variables}

Sociodemographic and clinical data concerning the severity of the cardiac disease (left ventricular ejection fraction (LVEF) and a history of myocardial infarction), as well as relevant cardiac risk factors (including smoking, hypertension, hypercholesterolemia, diabetes mellitus, history of stroke, peripheral atherosclerotic disease, history of cardiac disease other than myocardial infarction) were collected by the cardiologist during the admission to hospital for the myocardial infarction. LVEF was determined by echocardiography according to the modified Simpson's rule. ${ }^{19}$ Information on possible treatment with percutaneous coronary intervention (PCI) and assignment for cardiac rehabilitation after admission was retrieved from the hospital files.

\section{Psychiatric comorbidity}

Anxiety often coincides with depression in patients with myocardial infarction. ${ }^{20}$ Two meta-analyses have identified depression in individuals with myocardial infarction as a risk factor for all-cause mortality, cardiac mortality and cardiac events. $^{21,22}$ In order to adjust for depression as a covariate, depressive symptoms were measured with the BDI, a 21-item self-report questionnaire rating each item on a four-point Likert scale, which is well-validated in people with myocardial infarction. ${ }^{17,23}$

\section{Assessment of adverse outcomes}

The primary outcome was a major adverse cardiac event (MACE), defined by all-cause mortality or a readmission for a major cardiac event, occurring after the CAQ assessment at baseline or 4 months after discharge, respectively. Hospital readmissions with discharge diagnoses with ICD-9 codes 410, 411, 413, 414 (ischemic heart disease); 427.4 (ventricular fibrillation and flutter), 427.5 (cardiac arrest) and/or readmissions for an acute (unplanned) coronary intervention (coronary artery bypass graft/ percutaneous coronary intervention) were included as a major cardiac event. ${ }^{24}$ Data on all-cause mortality were obtained up until 1 January 2013 from the Dutch Central Bureau of Statistics by linkage to the municipal personal records database. Data concerning hospital admissions came from the Dutch national registry of hospital discharges and were obtained up until 1 January 2012 from the Dutch Central Bureau of Statistics by linkage to the municipal personal records database. Furthermore, in order to obtain more complete data, we also examined the administration and patient records of the Radboud University Medical Centre on readmissions for a MACE and/or mortality. These data were obtained up until 1 January 2013.

\section{Statistical analysis}

\section{Multiple imputation mode}

In order to use all available data for survival analysis, we performed multiple imputations in patients with data on a MACE. Rubin's rules were used to pool the data. ${ }^{25}$ Linear and logistic regression for multiple imputations was performed with all variables to be included in the final analysis model, as well as other relevant variables that were not included in the analyses, including cardiac risk and disease variables, PCI treatment and assignment to cardiac rehabilitation. These variables were examined for normal distribution and, if needed, transposed before the imputation was run; BDI was natural log-transformed. In total, $7.0 \%$ of all values in the imputation model were missing. A total of 67 data-sets were created, because $67.0 \%$ of the cases had at least one missing value. ${ }^{26}$ For each imputed file, SPSS estimated the missing values in 100 iterations. 


\section{Baseline}

Baseline demographic and clinical characteristics were compared between patients with and without a MACE with logistic regression (for categorical variables), Student's $t$-test, or when continuous variables were not normally distributed with the Mann-Whitney $U$-test.

\section{Correlation and survival analysis.}

The correlation between sum scores of the CAQ and the BDI was evaluated with Spearman's rho. For the survival analysis, Cox regression was used to evaluate risk of a MACE associated with CAQ sum scores at baseline and at 4 months after discharge. Next, using Cox regression, the association between each of the four CAQ subscales at baseline and at 4 months after discharge with MACE was evaluated. For analyses with CAQ at admission to hospital as determinant, the follow-up period for the primary end-point started at admission to hospital for the index myocardial infarction. For analyses with $\mathrm{CAQ}$ at 4 months after index myocardial infarction as determinant, the follow-up period for the primary end-point started at the date of this CAQ assessment. In all analyses the follow-up period for primary end-points ended on 1 January 2013, and patients who did not have the outcome of interest until 1 January 2013 were censored on 1 January 2013.

In the basic model, adjustments were made for age and gender. A priori we decided to adjust for a history of myocardial infarction and LVEF as objective parameters of cardiac disease severity, as both have been shown to be consistently related to worse cardiac prognosis. In addition, since cardiac-related hospital readmissions between baseline and 4 months also reflect cardiac disease severity, we adjusted for this characteristic when examining the CAQ score at 4 months after discharge. In the final model, we also adjusted for BDI scores.

\section{Sensitivity analyses}

Post hoc sensitivity analyses were performed in order to further adjust separately for possible confounders. In the basic model we tested those characteristics that at baseline differed between patients with and without a MACE. Furthermore, gender-effects were explored post hoc as depression has a greater impact on mortality in men than in women, ${ }^{27}$ although other studies have not found gender-specific effects with respect to cardiac prognosis. ${ }^{28,29}$ For the latter sensitivity analysis, we included this interaction variable in the regression model for multiple imputations. For all analyses, SPSS 20 for Windows was used and the significance level was set at 0.05 , two-tailed.

\section{Results}

\section{Sample}

Of 398 people with myocardial infarction admitted to the cardiology ward, 135 patients were, after a primary PCI, relocated to a hospital in their home area within 2 days of admission. Of the 263 patients who were still present at the time we asked for informed consent, 203 patients (77\%) agreed to participate. Ten individuals had to be excluded because of missing data on a MACE, leaving 193 patients to be analysed for the association between CAQ at baseline and a MACE. Baseline characteristics, resulting from the imputational model, for the 193 patients stratified according to occurrence of a MACE are shown in Table 1. Four people died before the assessment 4 months after discharge, leaving 189 people to be analysed for the association between CAQ at 4 months after discharge and a MACE.

The mean duration of assessment of CAQ and BDI at baseline was 3.7 days (s.d. $=3.8$ ) from admission to hospital (or index

\begin{tabular}{|c|c|c|c|c|c|c|}
\hline & $\begin{array}{l}\text { MACE } \\
(n=77)\end{array}$ & $\begin{array}{l}\text { No MACE } \\
(n=116)\end{array}$ & $\mathrm{OR}^{\mathrm{b}}$ & $t$-test & $\begin{array}{l}\text { Mann-Whitney } \\
\text { U-test }\end{array}$ & $P$ \\
\hline Age, mean (s.d.) & $66.0(11.6)$ & $58.7(11.0)$ & & 4.394 & & $<0.001^{* * *}$ \\
\hline Male, $n(\%)$ & $46(59.7)$ & $83(71.6)$ & 0.590 & & & 0.089 \\
\hline Stable relationship, $n$ (\%) & $57(74.0)$ & $97(83.6)$ & 1.791 & & & 0.107 \\
\hline Higher education, $n$ (\%) & $20(26.0)$ & $30(25.9)$ & 0.981 & & & 0.953 \\
\hline Hypertension, $n(\%)$ & $40(51.9)$ & $45(38.8)$ & 0.586 & & & 0.072 \\
\hline Diabetes mellitus, $n$ (\%) & $21(27.3)$ & $12(10.3)$ & 0.308 & & & $0.003^{* *}$ \\
\hline Hypercholesterolemia, $n$ (\%) & $22(28.6)$ & $33(28.4)$ & 0.994 & & & 0.985 \\
\hline History of myocardial infarction, $n(\%)$ & $41(53.2)$ & $21(18.1)$ & 0.194 & & & $<0.001^{* * *}$ \\
\hline $\begin{array}{l}\text { History of cardiac disease other than myocardial } \\
\text { infarction, }{ }^{c} n(\%)\end{array}$ & $30(39.0)$ & $11(9.5)$ & 0.164 & & & $<0.001^{* * *}$ \\
\hline Peripheral atherosclerotic disease, $n(\%)$ & $14(18.2)$ & $14(12.1)$ & 0.618 & & & 0.241 \\
\hline History of stroke, $n(\%)$ & $3(3.9)$ & $4(3.4)$ & 0.881 & & & 0.871 \\
\hline Smoker at inclusion, $n(\%)$ & $38(49.4)$ & $45(38.8)$ & 1.58 & & & 0.157 \\
\hline Family history of cardiac disease, $n$ (\%) & $34(44.2)$ & $51(44.0)$ & 0.993 & & & 0.982 \\
\hline Left ventricular ejection fraction \%, mean (s.d.) & $51.9(12.7)$ & $54.2(10.3)$ & & 1.164 & & 0.245 \\
\hline CAQ at hospital admission, mean (s.d.) & $1.5(0.6)$ & $1.3(0.6)$ & & 2.333 & & $0.020^{*}$ \\
\hline CAQ 4 months after discharge, mean (s.d.) & $1.3(0.7)$ & $1.0(0.6)$ & & 2.908 & & $0.004 * *$ \\
\hline BDI at hospital admission, median (IQR) & $6.8(4.0-12.0)$ & $6.0(3.9-9.5)$ & & & 3483 & 0.257 \\
\hline BDI 4 months after discharge, median (IQR) & $7.0(4.8-11.0)$ & $4.0(2.0-8.0)$ & & & 1758.5 & $0.002^{* *}$ \\
\hline Percutaneous coronary intervention, $n(\%)$ & $44(57.1)$ & $73(62.9)$ & 1.28 & & & 0.412 \\
\hline Assigned for cardiac rehabilitation, $n(\%)$ & $35(45.5)$ & $87(75.0)$ & 3.56 & & & $0.001 * *$ \\
\hline \multicolumn{7}{|c|}{$\begin{array}{l}\text { BDI, Beck Depression Inventory; CAQ, Cardiac Anxiety Questionnaire; IQR, interquartile range. } \\
\text { a. Rubin's rules were used to pool the data of the imputed data-sets.25 } \\
\text { b. We report odds ratio (based on univariate analysis) for all categorical variables. } \\
\text { C. Including for example stable angina pectoris, endocarditis, heart failure and atrial fibrillation. } \\
* P<0.05 \text {; } * * P<0.01 \text {; } * * * P<0.001 \text {. }\end{array}$} \\
\hline
\end{tabular}


myocardial infarction). The next CAQ assessment took place 138.8 days (s.d. $=59.4$ ) from admission to hospital (i.e. about 4 months post-myocardial infarction). A total of $77.8 \%$ of patients ( $n=147 / 189$ ) completed the CAQ at follow-up. Sum scores of CAQ and BDI were moderately correlated at admission to hospital (Spearman's $\rho=0.332, \quad P<0.001)$ ) and highly correlated at 4 months after discharge $(\rho=0.501, P<0.001)$.

\section{Predictors of a MACE}

During a mean follow-up period of 4.2 years (s.d. =2.0) (range 0-6.6 years), 77 (39.9\%) of 193 patients had a MACE after baseline. A total of 36 of them (46.8\%) died. During a mean follow-up period of 4.3 years (s.d. $=1.7)$ (range $0-6.0$ years), $67(35.4 \%)$ of 189 patients had a MACE after the 4-month CAQ assessment. A total of 32 of them $(47.8 \%)$ died. Patients who had an event during the follow-up period were older $(P<0.001)$, had higher CAQ scores at baseline $(P=0.020)$ and 4 months after the index myocardial infarction $(P=0.004)$, had higher BDI scores at 4 months after the index myocardial infarction $(P=0.002)$, were more likely to have a history of myocardial infarction and cardiac disease other than myocardial infarction $(P<0.001)$, or diabetes mellitus $(P=0.003)$ and were less likely to be assigned to cardiac rehabilitation $(P=0.004)$ (Table 1$)$.

\section{CAQ sum score and risk of a MACE}

CAQ at baseline was significantly associated with a higher risk of a MACE, independent of age and gender, LVEF and cardiac history, and BDI. CAQ at 4 months after discharge was also significantly associated with a higher risk of a MACE, even when adjusted for age, gender, LVEF, cardiac history, cardiac-related hospital readmissions between the index event and CAQ assessment and BDI (see Table 2 for hazard ratios (HRs) with 95\% confidence intervals).

\section{Subscales of CAQ at 4 months after discharge and risk of a MACE}

Only the subscale 'Avoidance' at 4 months after discharge was significantly associated with a MACE independent from all previously mentioned covariates. The prognostic association of 'Avoidance' at baseline lost significance after adjusting for severity of cardiac disease (see Table 3 for hazard ratios with 95\% confidence intervals).

\section{Sensitivity analyses for potential cardiac risk factors}

In sensitivity analyses, we tested whether characteristics that differed at baseline between patients with and without a MACE and that were not included in our model, might have influenced our results. Adding these variables (see Table 1) to model 2 generally did not affect the association between overall CAQ score and adverse prognosis. The only exception was the presence of a history of cardiac disease other than myocardial infarction: in this model the hazard ratio for CAQ at baseline and a MACE was attenuated to just be non-significant $(\mathrm{HR}=1.43,95 \%$ CI $0.96-2.11$, $P=0.076)$.

\section{Sensitivity analyses for gender differences in overall CAQ score and risk of a MACE}

We found an interaction effect between gender with overall CAQ score in the prognostic model (included variables: CAQ, gender, age, interaction gender-CAQ $)$ at baseline $\left(\mathrm{HR}_{\text {baseline }}=2.16,95 \%\right.$ CI 1.03-4.56, $P=0.043 ; \mathrm{HR}_{4 \text {-months }}=2.78,95 \%$ CI $1.00-7.72$, $P=0.050)$. Stratified analyses showed larger hazard ratios and more significant results in the women compared with the men. Only in women, did the full model remain significant for the CAQ at baseline (HR $=3.04,95 \%$ CI 1.47-6.28, $P=0.003, n=64$ of which $n=31$ had a MACE during follow-up), whereas the hazard ratio for the CAQ at 4 months lost significance after additional adjustment for BDI ( $\mathrm{HR}=2.17$ (95\% CI 0.78-6.07), $P=0.139, n=62$ of which $n=27$ had a MACE during follow-up, see online Tables DS1-3).

\section{Discussion}

\section{Main finding}

This study was the first to address the independent prognostic association of cardiac anxiety following myocardial infarction with adverse cardiac prognosis. Patients reporting higher cardiac anxiety were at increased risk of an adverse prognosis after adjustment for age, gender, cardiac disease severity parameters and depressive symptoms; with each point increase on the CAQ, which has a possible range of 0 to 4 , the risk of a new cardiac event increased from $56 \%$ (at baseline) to $71 \%$ (at 4 months after discharge). This effect seemed to be particularly driven by avoidance behaviour.

Our findings are in line with previous research reporting the prognostic impact of anxiety ${ }^{1}$ and anxiety disorders ${ }^{2,29,31}$ in patients with myocardial infarction. These heterogeneous studies used different anxiety measures and the reported hazard ratios in the meta-analysis (around 1.30) ${ }^{1}$ were based on dichotomous cut-off points of anxiety (present or not) that makes it difficult to compare the reported hazard ratios with ours. One study evaluated the prognostic association of separate dimensions of anxiety in patients with myocardial infarction. It also described an adverse prognostic association: significant associations for

Table 2 Hazard ratios ( $95 \% \mathrm{Cl}$ ) for a major adverse cardiac event (MACE) associated with scores on the Cardiac Anxiety Questionnaire (CAQ) during admission to hospital and at 4 months after discharge ${ }^{\text {a }}$

\begin{tabular}{|c|c|c|c|c|c|c|}
\hline & \multicolumn{3}{|c|}{ CAQ sum score at hospital admission $(n=77 / 193)$} & \multicolumn{3}{|c|}{ CAQ sum score at approximately 4 months after discharge $(n=67 / 189)$} \\
\hline & Hazard ratio $(95 \% \mathrm{Cl})$ & $P$ & $R^{2}, \%$ & Hazard ratio $(95 \% \mathrm{Cl})$ & $P$ & $R^{2}$ \\
\hline Model 1 & $1.70(1.16-2.46)$ & $0.006^{* *}$ & 4.1 & $2.09(1.38-3.17)$ & $0.001 * *$ & 8.0 \\
\hline Model 2 & $1.64(1.14-2.38)$ & $0.008^{* *}$ & 13.1 & $2.04(1.30-3.19)$ & $0.002^{\star *}$ & 12.9 \\
\hline Model 3 & $1.52(1.03-2.23)$ & $0.034^{*}$ & 22.6 & $2.00(1.24-3.23)$ & $0.005^{* *}$ & 20.3 \\
\hline Model 4 & 1.59 (1.04-2.43) & $0.033^{\star}$ & 22.8 & $1.77(1.04-3.02)$ & $0.036^{*}$ & 21.1 \\
\hline \multicolumn{7}{|c|}{ 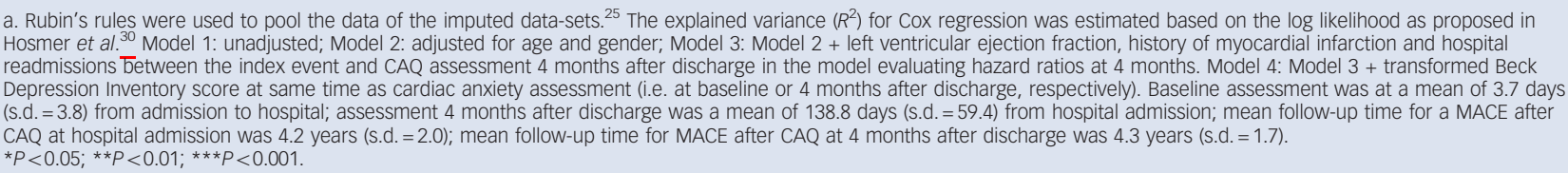 } \\
\hline
\end{tabular}




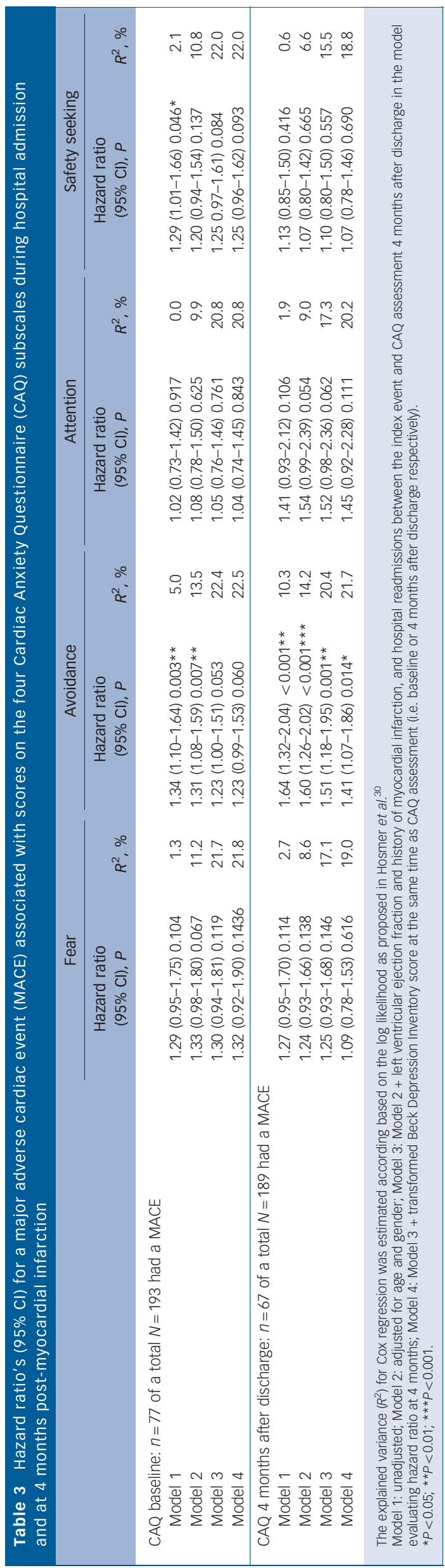

somatic anxiety $(\mathrm{HR}=1.29)$ and total anxiety $(\mathrm{HR}=1.38)$, with a trend for psychological anxiety. ${ }^{32}$ However, these anxiety dimensions were derived from a questionnaire assessing general anxiety (Hamilton Anxiety and Depression Rating Scales) and all dimensions are more or less associated with avoidance behaviour. These general findings are extended by our findings showing the most specific results for avoidance behaviour related to cardiac stimuli, especially at 4 months after discharge.

In our study, after adjustment for parameters of cardiac disease severity, the association of cardiac anxiety with a MACE remained significant. More importantly, the hazard ratios hardly attenuated, implying a prognostic effect of cardiac anxiety independent from cardiac disease severity. Adjustment for depressive symptoms did not affect the effect size of the cardiac anxiety on adverse cardiac prognosis. This is consistent with other studies assessing the association between general anxiety or anxiety disorder and cardiovascular events independent from depression. . $^{231,33}$

\section{Potential mechanisms}

The association between anxiety and ischemic heart disease might be explained by both behavioural and biological mechanisms. First, anxiety appears to be associated with unhealthy behaviour (for example, physical inactivity and smoking) in individuals at risk of coronary heart disease. ${ }^{34}$ Our finding that the main prognostic effect of cardiac anxiety was driven by the subscale assessing avoidance of physical activity supports this. Surprisingly though, in our study patients reporting higher anxiety less often smoked. Although this is in contrast with a recent finding suggesting less adherence to risk-reducing recommendations (such as cessation of smoking) in patients with myocardial infarction who are also anxious, ${ }^{35}$ this difference might be caused by the type of anxiety (general $v$. cardiac) examined, or by differences in coping strategies of individual patients.

Unfortunately, our data do not provide any insight into potential biological mechanisms explaining the negative association between anxiety and cardiac disease, such as reduced heart rate variability, cardiac arrhythmias and increased platelet activity or inflammation. ${ }^{36-38}$ For example, increased anxiety levels may trigger ventricular tachycardias in a dose-dependent way and are, among patients with implantable cardioverter defibrillators, associated with a higher number of implantable cardioverter defibrillator shocks. ${ }^{39}$

\section{Methodological considerations}

A strength of the present study is that cardiac anxiety was assessed not only at admission to hospital but also at 4 months after the infarction, when the possible inflating impact of the stressful admission to hospital and the event itself on anxiety parameters is expected to have settled down. ${ }^{15}$ Importantly, although our sample size was relatively small, it was large enough to adjust for several important confounders simultaneously. Nonetheless, we were not able to address all indicators of cardiac disease severity. Therefore, we adjusted for the two most important indicators, LVEF and a history of a myocardial infarction, in line with previous studies on this topic. However, since cardiac anxiety was inversely correlated with cardiac disease severity, as previously reported by our group, ${ }^{40}$ it seems unlikely that our results are driven by residual confounding because of cardiac disease severity.

In line with previous studies we chose as primary outcome a combination of all-cause mortality and cardiac readmissions. However, whereas previous studies included a rather broad range of cardiovascular events, ${ }^{1,2,21}$ we only included readmissions for 
acute ischemic events. We consider this as an advantage. As patients with high cardiac anxiety might be more likely to consult their doctor and be admitted to the hospital with cardiac complaints, this might partly explain the association between cardiac anxiety and cardiovascular-related hospital readmissions. By only including readmissions because of acute ischemic events, this risk is minimised.

Although the prognostic association of cardiac anxiety with a MACE was particularly driven by avoidance of physical activity, it is important to realise that physical activity was not assessed in the present study and some of the other dimensions of cardiac anxiety (fear and attention, respectively) showed even higher hazard ratios than avoidance. The prognostic associations of these subscales were not significant in the final model, but this may be explained by a power problem (type two error). Studies in larger populations are needed to examine this more closely. Future studies should include physical activity as a mediating mechanism when studying the prognostic impact of cardiac anxiety.

Limitations of the present study include the absence of information on presence (and/or history) of a formal diagnosis of an anxiety and/or depressive disorder, nor did we have information on possible psychiatric treatment. Therefore, we do not know how cardiac anxiety is related to psychiatric diagnosis. As (some) anxiety disorders have been associated with the development of coronary heart disease in the general population ${ }^{12,41}$ and prognosis in patients with heart disease, ${ }^{2,29,30}$ this would have been interesting. Nevertheless, we did adjust for depressive symptoms, which is an important possible confounder. ${ }^{21,22}$

Our study had a good response rate of $77 \%,{ }^{15}$ even though we had to exclude the most severely ill patients. On the other hand, patients with milder symptoms, who were transported to other hospitals within 2 days, were not eligible for the present study and it is questionable whether this limits generalisation of our results.

In our study, the association between cardiac anxiety and adverse prognosis could not be explained by cardiac disease severity parameters, including LVEF and cardiac history. Nevertheless, it is still possible that the association is confounded by disease severity, especially since some symptoms of cardiac anxiety, such as chest pain, might also be symptoms of heart disease. However, most items in the CAQ focus on the impact of these symptoms on affect (anxious), thought (worrying) or behaviour (avoidance and safety-seeking behaviour).

Although the hazard ratios were particularly higher for women, the gender-specific findings should be considered preliminary because of lack of statistical power. Moreover, the gender-effect was opposite to that found for the association between depression and mortality in cardiac patients ${ }^{27}$ and two smaller studies with underrepresentation of women did not find gender effects. ${ }^{28,29}$ Therefore, future studies examining anxiety should preferably examine possible interaction effects with gender.

\section{Clinical implications}

The present findings of a prognostic association with a MACE, independent from cardiac disease severity and depressive symptoms, stress the potential clinical impact of cardiac anxiety. Interestingly, when looking at the CAQ score alone, the explained variance increased in the follow-up model compared with the baseline measurement (4 and $8 \%$ respectively), indicating the importance of identifying (persisting) cardiac anxiety symptoms in the months post-myocardial infarction. Diagnosing elevated cardiac anxiety symptoms, and its specific subtypes, can be helpful in developing specific interventions to reduce maintaining or exacerbating factors, such as avoidance of cardiac stimuli and physical exercise. Cognitive-behavioural therapy (CBT) may target anxiety-related avoidance behaviour, which may even result in a better cardiac outcome. General CBT focusing on stress management has been shown to improve cardiac outcome over 8 years post-myocardial infarction. ${ }^{42}$ For this reason, cardiac anxiety should be explicitly addressed in cardiac rehabilitation programmes. Whereas there is awareness of general anxiety in cardiac rehabilitation programmes and recent studies have paid attention to specific types of anxiety recognised by the DSM, such as $\mathrm{GAD},{ }^{9}$ cardiac anxiety may in fact be relatively unexplored. Outcomes of cardiac rehabilitation programmes might even improve further if physical exercise is used as a behavioural experiment to test out specific cardiac fears patients may have. In conclusion, the findings of the present study indicate the need to evaluate and target cardiac anxiety - in addition to depression and general anxiety - in the future. Medical Centre, Nijmegen, The Netherlands; Marij Zuidersma, PhD, University Medical Center Groningen, University Center for Psychiatry, and Interdisciplinary Center for Psychopathology and Emotion Regulation, University of Groningen, Groningen, The Netherlands; Martijn Lappenschaar, MD, PhD, Department of Psychiatry, Radboud University Medical Centre, Nijmegen, The Netherlands: Gheorghe Pop, MD, PhD, Department of Cardiology, Radboud University Medical Centre Nijmegen, Nijmegen, The Netherlands; Annelieke M. Roest, PhD, University Medical Center Groningen, University Center for Psychiatry, and Interdisciplinary Center for Psychopathology and Emotion Regulation, University of Groningen, Groningen, The Netherlands; Anton J. L. M. Van Balkom, MD, PhD, Department of Psychiatry and EMGO+ Institute, VU University Medical Center, GGZinGeest, Amsterdam, The Netherlands; Anne E. M. Speckens, MD, PhD, Department of Psychiatry, Radboud University Medical Centre, Nijmegen, The Netherlands; Richard C. Oude Voshaar, MD, PhD, University Medical Center Groningen, University Center for Psychiatry, and Interdisciplinary Center for Psychopathology and Emotion Regulation, University of Groningen, Groningen, The Netherlands.

Correspondence: Maria H. C. T. van Beek, Department of Psychiatry, Radboud University Medical Center, Nijmegen, The Netherlands. Email: marleen.vanbeek@radboudumc.nl

First received 30 Aug 2015, final revision 22 Apr 2016, accepted 23 Apr 2016

\section{References}

1 Roest AM, Martens EJ, Denollet J, De Jonge P. Prognostic association of anxiety post myocardial infarction with mortality and new cardiac events: a meta-analysis. Psychosom Med 2010; 72: 563-9.

2 Roest AM, Zuidersma M, de Jonge P. Myocardial infarction and generalised anxiety disorder: 10-year follow-up. Br J Psychiatry 2012; 200: 324-9.

3 Versteeg H, Hoogwegt MT, Hansen TB, Pedersen SS, Zwisler AD, Thygesen LC. Depression, not anxiety, is independently associated with 5-year hospitalizations and mortality in patients with ischemic heart disease. J Psychosom Res 2013; 75: 518-25.

4 Lane D, Carroll D, Ring C, Beevers DG, Lip GY. Mortality and quality of life 12 months after a myocardial infarction: effects of depression and anxiety. Psychosom Med 2001; 63: 221-30.

5 Mayou RA, Gill D, Thompson DR, Day A, Hicks N, Volmink J, et al. Depression and anxiety as predictors of outcome after myocardial infarction. Psychosom Med 2000; 62: 212-19.

6 Parker GB, Owen CA, Brotchie HL, Hyett MP. The impact of differing anxiety disorders on outcome following an acute coronary syndrome: time to start worrying? Depress Anxiety 2010; 27: 302-9.

7 Parker G, Hyett M, Hadzi-Pavlovic D, Brotchie H, Walsh W. GAD is good? Generalized anxiety disorder predicts a superior five year outcome following an acute coronary syndrome. Psychiatry Res 2011; 188: 383-9.

8 Meyer T, Hussein S, Lange HW, Herrmann-Lingen C. Anxiety is associated with a reduction in both mortality and major adverse cardiovascular events five years after coronary stenting. Eur J Prev Cardiol 2015; 22: 75-82.

9 Tully PJ, Winefield HR, Baker RA, Denollet J, Pedersen SS, Wittert GA, et al. Depression, anxiety and major adverse cardiovascular and cerebrovascular events in patients following coronary artery bypass graft surgery: a five year longitudinal cohort study. Biopsychosoc Med 2015; 9: 14.

10 Laan W, Termorshuizen F, Smeets HM, Boks MP, de Wit NJ, Geerlings MI. A comorbid anxiety disorder does not result in an excess risk of death among patients with a depressive disorder. J Affect Disord 2011; 135: 284-91. 
11 Batelaan NM, ten Have M, Van Balkom AJ, Tuithof M, de Graaf R. Anxiety disorders and onset of cardiovascular disease: The differential impact of panic, phobias and worry. J Anxiety Disord 2014; 28: 252-8.

12 Albert CM, Chae CU, Rexrode KM, Manson JE, Kawachi I. Phobic anxiety and risk of coronary heart disease and sudden cardiac death among women. Circulation 2005; 111: 480-7.

13 Eifert GH, Thompson RN, Zvolensky MJ, Edwards K, Frazer NL, Haddad JW, et al. The Cardiac Anxiety Questionnaire: development and preliminary validity. Behav Res Ther 2000; 38: 1039-53.

14 Van Beek MH, Oude Voshaar RC, Deelen van FM, Van Balkom AJ, Pop G, Speckens AE. The Cardiac Anxiety Questionnaire: cross-validation among cardiac inpatients. Int J Psychiatry Med 2012; 43: 349-64.

15 Van Beek MH, Mingels M, Oude Voshaar RC, Van Balkom AJ, Lappenschaar M, Pop G, et al. One-year follow up of cardiac anxiety after a myocardial infarction: a latent class analysis. J Psychosom Res 2012; 73: 362-8.

16 Kaptein $\mathrm{KI}$, de Jonge $\mathrm{P}$, van den Brink $\mathrm{RH}$, Korf J. Course of depressive symptoms after myocardial infarction and cardiac prognosis: a latent class analysis. Psychosom Med 2006; 68: 662-8.

17 Beck AT, Ward $\mathrm{CH}$, Mendelson M, Mock J, Erbaugh J. An inventory for measuring depression. Arch Gen Psych 1961; 4: 561-71.

18 Marker CD, Carmin CN, Ownby RL. Cardiac anxiety in people with and without coronary atherosclerosis. Depress Anxiety 2008; 25: 824-31.

19 Folland ED, Parisi AF, Moynihan PF, Jones DR, Feldman CL, Tow DE. Assessment of left ventricular ejection fraction and volumes by real-time two-dimensional echocardiography. A comparison of cineangiographic and radionuclide techniques. Circulation 1979; 60: 760-6.

20 Denollet J, Strik JJ, Lousberg R, Honig A. Recognizing increased risk of depressive comorbidity after myocardial infarction: looking for 4 symptoms of anxiety-depression. Psychother Psychosom 2006; 75: 346-52.

21 Meijer A, Conradi HJ, Bos EH, Thombs BD, van Melle JP, de Jonge P. Prognostic association of depression following myocardial infarction with mortality and cardiovascular events: a meta-analysis of 25 years of research. Gen Hosp Psychiatry 2011; 33: 203-16.

22 Nicholson A, Kuper $H$, Hemingway $H$. Depression as an etiologic and prognostic factor in coronary heart disease: a meta-analysis of 6362 events among 146538 participants in 54 observational studies. Eur Heart J 2006; 27: 2763-74.

23 Strik JHM, Honig A, Lousberg R, Denollet J. Sensitivity and specificity of observer and self-report questionnaires in major and minor depression following myocardial infarction. Psychosomatics 2001; 42: 423-8.

24 World Health Organization. International Statistical Classification of Diseases and Related Health Problems (ICD-9). WHO, 1978.

25 Rubin DB. Multiple Imputation for Nonresponse in Surveys. Wiley, 1987

26 White IR, Royston P, Wood AM. Multiple imputation using chained equations: issues and guidance for practice. Stat Med 2011; 30: 377-99.

27 Van Loo HM, van den Heuvel ER, Schoevers RA, Anselmino M, Carney RM, Denollet J, et al. Sex dependent risk factors for mortality after myocardial infarction: individual patient data meta-analysis. BMC Med 2014; 12: 242.
28 Frasure-Smith N, Lesperance F, Juneau M, Talajic M, Bourassa MG. Gender, depression, and one-year prognosis after myocardial infarction. Psychosom Med 1999; 61: 2637.

29 Frasure-Smith N, Lesperance F. Depression and anxiety as predictors of 2-year cardiac events in patients with stable coronary artery disease. Arch Gen Psychiatry 2008; 65: 62-71.

30 Hosmer DW, Lemeshow S, May M. Applied Survival Analysis: Regression Modelling of Time-to-Event Data (2nd edn). Wiley, 2008

31 Martens EJ, de Jonge P, Na B, Cohen BE, Lett H, Whooley MA. Scared to death? Generalized anxiety disorder and cardiovascular events in patients with stable coronary heart disease: The Heart and Soul Study. Arch Gen Psychiatry 2010; 67: 750-8.

32 Roest AM, Martens EJ, de Jonge P, Denollet J. Symptom dimensions of anxiety following myocardial infarction: associations with depressive symptoms and prognosis. Health Psychol 2014; 33: 1468-76.

33 Strik JJ, Denollet J, Lousberg R, Honig A. Comparing symptoms of depression and anxiety as predictors of cardiac events and increased health care consumption after myocardial infarction. J Am Coll Cardiol 2003; 42: 1801-7.

34 Bonnet F, Irving K, Terra JL, Nony P, Berthezene F, Moulin P. Anxiety and depression are associated with unhealthy lifestyle in patients at risk of cardiovascular disease. Atherosclerosis 2005; 178: 339-44.

35 Kuhl EA, Fauerbach JA, Bush DE, Ziegelstein RC. Relation of anxiety and adherence to risk-reducing recommendations following myocardial infarction. Am J Cardiol 2009; 103: 1629-34.

36 Cameron OG, Smith CB, Lee MA, Hollingsworth PJ, Hill EM, Curtis GC. Adrenergic status in anxiety disorders: platelet alpha 2-adrenergic receptor binding, blood pressure, pulse, and plasma catecholamines in panic and generalized anxiety disorder patients and in normal subjects. Biol Psychiatry 1990; 28: 3-20

37 Pitsavos C, Panagiotakos DB, Papageorgiou C, Tsetsekou E, Soldatos C, Stefanadis C. Anxiety in relation to inflammation and coagulation markers, among healthy adults: the ATTICA study. Atherosclerosis 2006; 185: 320-6.

38 Martens EJ, Nyklícek I, Szabó BM, Kupper N. Depression and anxiety as predictors of heart rate variability after myocardial infarction. Psychol Med 2008; 38: 375-83

39 Habiboviæ M, Pedersen SS, van den Broek KC, Theuns DA, Jordaens L, van der Voort $\mathrm{PH}$, et al. Anxiety and risk of ventricular arrhythmias or mortality in patients with an implantable cardioverter defibrillator. Psychosom Med 2013; 75: 36-41.

40 van Beek MH, Oude Voshaar RC, van Deelen FM, van Balkom AJ, Pop G, Speckens AE. Inverse correlation between cardiac injury and cardiac anxiety: a potential role for communication. J Cardiovasc Nurs 2014; 29: 448-53.

41 Janszky I, Ahnve S, Lundberg I, Hemmingsson T. Early-onset depression, anxiety, and risk of subsequent coronary heart disease: 37-year follow-up of 49,321 young Swedish men. J Am Coll Cardiol 2010; 56: 31-7.

42 Gulliksson M, Burell G, Vessby B, Lundin L, Toss H, Svärdsudd K. Randomized controlled trial of cognitive behavioral therapy vs standard treatment to prevent recurrent cardiovascular events in patients with coronary heart disease: Secondary Prevention in Uppsala Primary Health Care project (SUPRIM). Arch Intern Med 2011; 171: 134-40. 